\title{
Prevalence of syphilis among patients living with HIV treated in Bialystok HIV/AIDS Center
}

\author{
Joanna Pogorzelska, Anna Grzeszczuk, Alicja D. Wandałowicz, Robert Flisiak \\ Department of Infectious Diseases and Hepatology, Medical University of Bialystok, Poland
}

\begin{abstract}
Introduction: In recent years, the incidence of syphilis rise globally. Men who have sex with men (MSM) with human immunodeficiency virus (HIV) infection are at higher risk for syphilis coinfection than general population.

Material and methods: This study evaluated the prevalence and clinical presentation of Treponema pallidum infection among adult people living with HIV (PLHIV) in North-Eastern Poland, between 2014-2016. Venereal disease research laboratory (VDRL) titer test for syphilis was performed as a screening. Fluorescent treponemal antibody (FTA), fluorescent treponemal antibody absorption (FTA-ABS), and Treponema pallidum hemagglutination assay (TPHA) were used as confirmatory tests. Results: Two hundred and fifty HIV-infected patients participated in the study, including 25 MSM (10\%). VDRL was positive in 15 patients (14 males and 1 female), and in 12 of them confirmatory tests were positive ( $4.8 \%$ tested). All of them were male, with median age of 41 years. Syphilis was diagnosed in 8 of homosexual and/or bisexual, which is 32\% of HIV-infected MSM patients. Only 5 patients $(62 \%)$ with HIV and syphilis coinfection demonstrated clinical symptoms: tongue and hard palate lesions, pharyngitis with exanthema on hands and feet, penile primary chancre, and alopecia areata.

Conclusions: We demonstrated high prevalence of syphilis and asymptomatic course of the disease among HIV-positive MSM patients in North-Eastern Poland, that stress the necessity of regular syphilis testing in this population. Early diagnosis and proper treatment of syphilis is crucial to reduce spread of this infection.
\end{abstract}

HIV AIDS Rev 2017; 16, 4: 265-268 DOI: https://doi.org/10.5114/hivar.2017.72028

Key words: syphilis, human immunodeficiency virus, North-Eastern Poland.

\section{Introduction}

The incidence of syphilis infection continues to rise globally [1]. In Western and Eastern Europe, men who have sex with men (MSM) and especially human immunodeficiency virus (HIV)-positive MSM are disproportionately affected [2]. In 2015 in Poland, there were 1,338 reported cases of all types of syphilis, which was higher by 126 cases reported in 2014 .
In 2016, the number of syphilis infection increased to 1,596 cases, what gives incidence rate of 4.15/100,000 inhabitants. In Podlaskie Voivodship, North-Eastern Poland, 13 cases were reported in 2015 and 27 cases in 2016, with incidence rate of 2.27/100,000 inhabitants [3,4]. Treponema pallidum and HIV share the same route of transmission. Syphilitic ulcers may increase the risk of infection with HIV, and HIV can alter the natural course of syphilis [5-7].

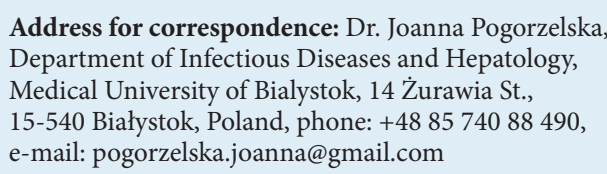

Address for correspondence: Dr. Joanna Pogorzelska Department of Infectious Diseases and Hepatology, Medical University of Bialystok, 14 Żurawia St., 15-540 Białystok, Poland, phone: +48 8574088 490, e-mail: pogorzelska.joanna@gmail.com

Article history:

Received: 03.11.2017

Received in revised form: 26.11.2017

Accepted: 26.11.2017

Available online: 30.11 .2017
International Journal of HIV-Related Problems

HIV \& AIDS

R e v i e w 
The aim of this study was to determine the prevalence of Treponema pallidum infection among people living with HIV (PLHIV), treated in HIV/AIDS treatment center in Bialystok. Moreover, clinical, socio-demographic and epidemiologic characteristics of co-infected patients were described.

\section{Material and methods}

The cross-sectional study of syphilis prevalence was conducted among adult people living with HIV (PLHIV) treated at the Department of Infectious Disease and Hepatology, and associated HIV/AIDS out-patient, Medical University of Bialystok, Poland between 2014 and 2016.

Venereal disease research laboratory (VDRL) test was performed as a screening for syphilis and fluorescent treponemal antibody (FTA), fluorescent treponemal antibody absorption (FTA-ABS), and Treponema pallidum hemagglutination assay (TPHA) were used as confirmatory tests.

Age, sex, length, and way of HIV infection were analyzed. Moreover, clinical signs and symptoms, history of previous STI in syphilis seroactive patients were evaluated.

Data were expressed as the median, percentages as well as an interquartile range.

\section{Results}

Two hundred and fifty PLHIV, 182 male and $68 \mathrm{fe}-$ male, aged 40.8 years (min-max: 20-72) were included in the study (Table 1). VDRL test was positive in 15 patients, that included 14 males and 1 female. In 12 of those patients, the confirmatory tests were positive, allowing the syphilis diagnose in $4.8 \%$ of the whole studied PLHIV. In 3 cases, VDRL results were shown to be false positive (one female and two males). In one male patient, with acute retroviral infection, the syphilis was diagnosed based on a medical history regardless of initially negative result of VDRL test, because the syphilis confirmatory tests were performed and were positive, allowing the diagnosis of early syphilis (FTA, FTA-ABS, TPHA - positive).

All $12 \mathrm{HIV} /$ syphilis co-infected patients were male (Table 2). In 8 patients, the route of infection was homo-

Table 1. Characteristics of study population

\begin{tabular}{l|c}
\hline Factor & Patients with HIV \\
\hline \begin{tabular}{l} 
Gender, $n(\%)$ \\
\cline { 2 - 2 } Male
\end{tabular} & $182(73)$ \\
\hline Female & $68(27)$ \\
\hline Median age, years (min/max) & $40.8(20 / 72)$ \\
\hline \begin{tabular}{l} 
Route of HIV infection, $n(\%)$ \\
\hline IVDU
\end{tabular} \\
\hline HTR & $136(54)$ \\
\hline MSM & $89(36)$ \\
\hline
\end{tabular}

IVDU - intra-venous drug use, HTR - heterosexual, MSM - man who have sex with man sexual (MSM), while remaining 4 patients denied homosexual intercourses.

Among those HIV/syphilis co-infected patients, 5 demonstrated clinical symptoms. Primary chancres were seen in 3 patients, one penile ulcer (Fig. 1), and the other 2 with early syphilis had exanthema on hands and feet. The manifestations of late syphilis observed among our patients included: tongue and hard palate lesions (Fig. 2), pharyngitis with exanthema on hands and feet, and alopecia areata.

In three patients with HIV/syphilis co-infection, lumbar puncture (LP) was performed. Other individuals were free of neurological symptoms and refused consent for LP. Among those with performed LP, cerebrospinal fluid (CSF) was normal in one patient, whereas 2 other patients showed presence of FTA titers in CSF. Finally, neurosyphilis was diagnosed in one patient, and confirmed by positive FTA and TPHA tests as well as increased number of mononuclear cells in CSF. Moreover, one of HIV/syphilis co-infected patient had condylomata acuminata, and 3 patients had $\mathrm{HCV}$ infection at the time of syphilis diagnosis. Syphilis and HIV infection was diagnosed at the same time in 2 patients. Three out of $12 \mathrm{HIV} /$ syphilis co-infected patients received antiretroviral therapy with achieved undetectable HIV RNA. Only 3 out of $12 \mathrm{HIV} /$ syphilis co-infected patients received intravenous penicillin (benzylpenicillin) treatment, whereas remaining 9 were treated with oral doxycycline.

\section{Discussion}

Since 1990's, the incidence of syphilis has been growing among PLHIV, especially in young, homosexual, and

Table 2. Characteristics of patients with HIV and syphilis coinfection

\begin{tabular}{|c|c|}
\hline Factor & Patients with HIV \\
\hline \multicolumn{2}{|l|}{ Gender, $n(\%)$} \\
\hline Male & $12(100)$ \\
\hline Female & $0(0)$ \\
\hline Median age, years (min/max) & $41(29.5-52.0)$ \\
\hline \multicolumn{2}{|l|}{ Route of HIV infection, $n$ (\%) } \\
\hline IVDU & $3(25)$ \\
\hline HTR & $1(8)$ \\
\hline MSM & $5(42)$ \\
\hline Bisexual & $3(25)$ \\
\hline Length of HIV infection (IQR) & $7.5(5)$ \\
\hline Median CD4 cell count, cells/ $\mu$ l(IQR) & $378(286)$ \\
\hline Median HIV-1 RNA, copies/ml (IQR) & $155,310.4(43,978)$ \\
\hline Patients on HAART, $n(\%)$ & $8(66.7)$ \\
\hline HIV VL $<20, n(\%)$ & $3(25)$ \\
\hline
\end{tabular}




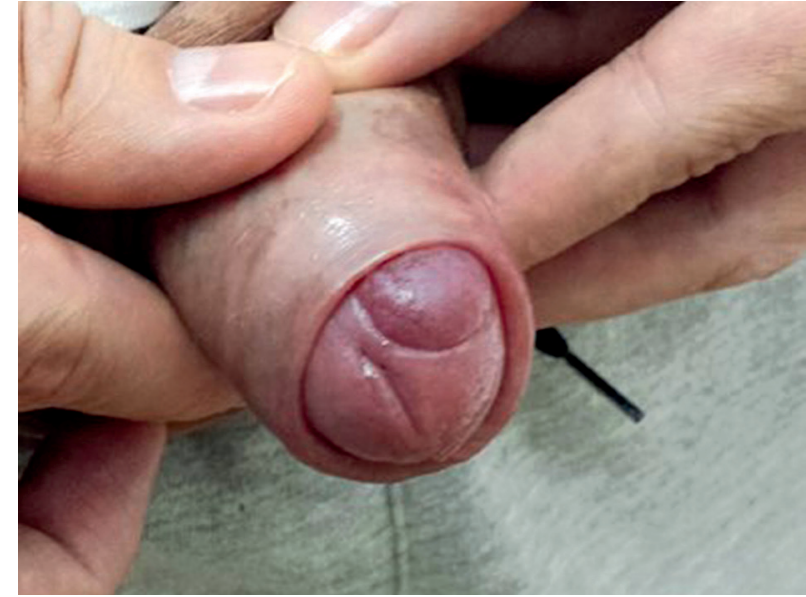

Fig. 1. Penile primary chancre

bisexual individuals $[8,9]$. Among 250 PLHIV included in the study, syphilis was diagnosed in 12 individuals, which seem to be quite low prevalence. However, MSM constitute only $10 \%$ of our study group and syphilis was diagnosed in 8 of them, which is a quite high prevalence of $32 \%$. This observation is in line with a German study, where the prevalence of HIV infection among patients with newly diagnosed syphilis was as high as $45 \%$ [5].

Several studies show that HIV co-infection can modify the course of syphilis infection. Manifestations of syphilis in PLHIV is thought to differ from that in HIV-negative patients. Lack of immunologic control may cause multiple primary lesions, slower resolution of primary chancres, and poor expression of symptoms in secondary syphilis [10]. HIV and syphilis co-infected patients may have more rapid progression to tertiary manifestations, delay or failure of titer decline following treatment, higher rate of serologic failure, and relapse $[11,12]$.

In HIV-positive patients, unusual manifestations and fulminant progress of syphilis are often observed [13]. Reactivation of earlier infections as well as shorter latency periods and faster progression to the later stages, occur in addition to symptoms of the coexistent stages. Neurosyphilis can be diagnosed in about $20 \%$ of syphilis/HIV co-infected patients during the early syphilis stages [10]. In our study, only 5 out of 12 patients with HIV and syphilis co-infection had clinical manifestations observed. Primary chancres were observed in 3 patients, and late syphilis manifestation in 2 patients. Neurosyphilis was diagnosed in 1 patient. Observations carried out in Warsaw showed very high percentage of neurosyphilis among HIV-positive patients with early syphilis. Lumbar puncture was performed in 72 patients, and neurosyphilis was confirmed in 65 cases (90.3\%) [10].

In our study, three out of 12 patients with HIV/syphilis co-infection were receiving ART therapy resulting with viral suppression. Syphilis can lead to a temporary increase in HIV viral load, and to an additional deterioration of the immune status even in patients on effective ART [5].

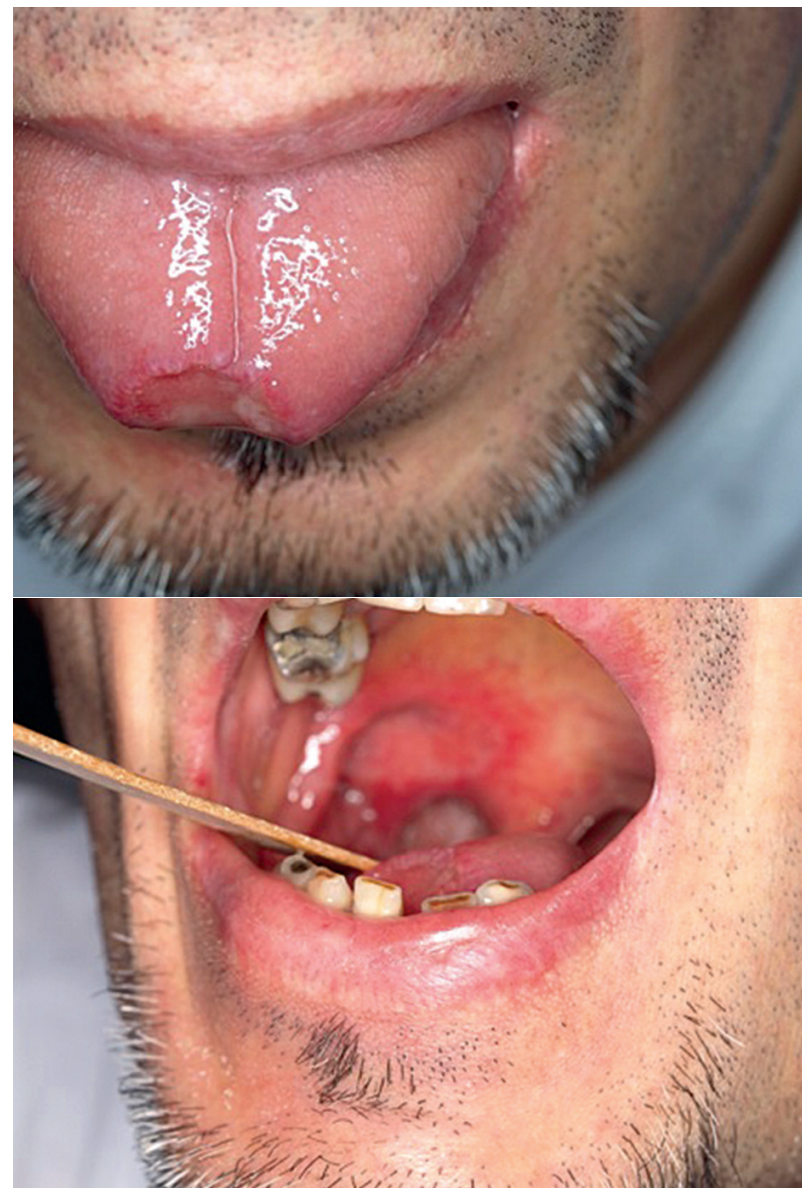

Fig. 2. Oral manifestation of late syphilis, tongue lesion, and hard palate lesions

The incidence of sexually transmitted infections (STIs) increase more rapidly among HIV-infected patients. Other STDs, like human papilloma virus infection, is one of the most frequently transmitted viral infection. One of our HIV/syphilis co-infected patient had condylomata acuminata, and three patients had HCV co-infection due to history of intravenous drug use. On the other hand, patients with STDs should be tested for HIV, but this policy is cost effective, which had recently been proven in HIDES I and II studies [14-16].

Penicillin remains the treatment of choice for syphilis, with preserved sensitivity and the best efficacy recorded [5]. Regardless of the penicillin treatment recommendations of both international and Polish HIV/syphilis treatment guidelines, only 3 of our HIV/syphilis co-infected patients received penicillin intravenously [17]. The main reason for such a discrepancy between guidelines and our real life practice was the lifestyle of the treated patients with no possibility of their hospitalization due to penicillin administration, which is reserved only for hospitals. Our nine remaining patients were treated with oral doxycycline, some with considerable time delay since syphilis diagnosis. All observed patients were successfully treated. 


\section{Conclusion}

High prevalence of syphilis among HIV-positive MSMs demonstrated in North-Eastern Poland stress out the necessity of regular syphilis testing in this population.

\section{Conflict of interest}

The authors declare no potential conflicts of interest with respect to the research, authorship, and/or publication of this article.

\section{References}

1. Harrison LW. Principles of Internal Medicine. $17^{\text {th }}$ ed. McGraw-Hill Companies, 2008.

2. Rowley D, Swiecki P, Firlag-Burkacka E, et al. Clinical and epidemiological characteristics of patients with early syphilis from three academic centres in Poland, Germany, and Ireland: initial findings from the POETS study. Sex Transm Infect 2015; 91: 389-394.

3. Majewski S, Rudnicka I. Sexually transmitted diseases in Poland in 2011. Przegl Epidemiol 2013; 67: 283-286.

4. http://wwwold.pzh.gov.pl/oldpage/epimeld/2015/Ch_2015.pdf

5. www.hivbook.com

6. Pialoux G, Vimont S, Moulignier A, et al. Effect of HIV infection on the course of syphilis. AIDS Rev 2008; 10: 85-92.

7. Kenyon C, Osbak KK, Crucitti T, et al. The immunological response to syphilis differs by HIV status; a prospective observational cohort study. BMC Infect Dis 2017; 17: 111.

8. Beyrer C, Baral S, Walker D, et al. The expanding epidemics of HIV-1 among men who have sex with men in low and middle income countries: diversity and consistency. Epidemiol Rev 2010; 32: 137-151.

9. Szetela B, Gasiorowski J. Rapid progression and overlapping of skin eruptions in a patient with secondary and tertiary syphilis co-infected with HIV. AIDS Res Hum Retroviruses 2016; 32: 874-875.

10. Firlag-Burkacka E, Swiecki P, Cielniak I, et al. High frequency of neurosyphilis in HIV-positive patients diagnosed with early syphilis. HIV Med 2016; 17: 323-326.

11. Serwin AB, Koper M, Unemo M. Clinical and epidemiological characteristics of males with syphilis in Białystok, Poland. Przegl Epidemiol 2015; 69: 41-45.

12. Szetela B, Fleische K, Gasiorowski J, et al. Panuveitis as syphilis manifestation in HIV-1 infected patients. HIV \& AIDS Review 2007; 6: 23-25.

13. Gregory N, Sanchez M, Buchness MR. The spectrum of syphilis in patients with human immunodeficiency virus infection. J Am Acad Dermatol 1990; 22 (6 Pt 1): 1061-1067.

14. Kassutto S, Sax PE. HIV and syphilis coinfection: trends and interactions. AIDS Clin Care 2003; 15: 9-15.

15. Sullivan AK, Raben D, Reekie J, et al. Feasibility and effectiveness of indicator condition-guided testing for HIV: results from HIDES I (HIV indicator diseases across Europe study). PLoS One 2013; 8: e52845.

16. Raben D, Mocroft A, Rayment M, et al. Auditing HIV Testing Rates across Europe: Results from the HIDES 2 Study. PLoS One 2015; 10: e0140845.

17. http://www.eacsociety.org/files/guidelines_8.2-english 